ISBN 978-93-84468-80-4

International Conference on Recent Trends in Engineering and Technology

(RTET-2016))

Pattaya (Thailand) Dec. 14-16, 2016

\title{
Determining the Acute Inflammations using Back Propagation Algorithm with Adaptive Learning Coefficients
}

\author{
Humar Kahramanlı \\ Selcuk University Technology Faculty Department of Computer Engineering
}

\begin{abstract}
Artificial Neural Networks often finds application areas because of successful results in engineering, medicine and finance. Nevertheless, there are still many deficiencies and therefore ANN does not reach the desired level of success. Researchers have done a lot of work to reduce those deficiencies. In this study, the approach as Back Propagation Algorithm with Adaptive Learning coefficient is presented and was applied to determine acute inflammations.
\end{abstract}

Keywords: Artificial neural networks, back propagation algorithm, classification, acute inflammations.

\section{Introduction}

Artificial neural network (ANN) is a mathematical model which was inspired from the biological neural networks. ANN is a non-linear mapping model and has been successfully applied in many domains in data mining [1]. The reason for being commonly used is to present some properties such as learning from examples and exhibiting some capability for generalization beyond the training data [2].

ANN can be trained using many techniques. Gradient Descent (GD) algorithm is a widely used ANN training technique due to its inherent simplicity and ease of implementation. In GD, an error function computes the difference between the observed and predicted values and hill climbing or descent is used to find the weight values which reduce the error [1].

Back Propagation (BP) is based on GD algorithm, which was proposed by Rumelhart and McClelland [3]. $\mathrm{BP}$ is the most widely used algorithm for connectionist learning. It has ideal effects in pattern recognition, process control, fault diagnosis, function approximation and prediction because of its simple structure and the ability to approximate any continuous nonlinear function with arbitrary precision [4].

Seguritan et al. [5] have designed a method to predict phage structural protein using ANN. They trained ANNs to classify viral structural proteins using amino acid frequency; then they added estimates of protein isoelectric points as a feature to ANNs that classify specialized families of proteins, namely major capsid and tail proteins. These more specialized ANNs were more accurate than the structural ANNs. Kottaimalai et al. [6] have used Principal Component Analysis with Artificial Neural Network to train brain signals in order to classify the mental tasks. Rai et al [7]. have classified ECG signal data into two classes (abnormal and normal class) using three various neural classifiers and performances have compared. Kumar et al. [8] have used feed-forward backpropagation neural network for classification. Levenberg - Marquardt (LM) optimization technique has been used as training algorithm to update the weight and bias values.

In this study, the approach as BP algorithm with adaptive learning coefficient is presented and was applied to determine acute inflammations. 


\section{Materials and Methods}

\subsection{Back Propagation Algorithm}

The most commonly used training algorithm in applications is Back Propagation learning algorithm. It is a usually considered as supervised learning method. BP is a most preferred algorithm since it is an easy to understand and to prove mathematically [9]. The Back Propagation algorithm looks for the minimum of the error function in weight space using the method of gradient descent. The combination of weights which minimizes the error function is considered to be a solution of the learning problem. Since this method requires computation of the gradient of the error function at each iteration step, we must guarantee the continuity and differentiability of the error function [10].

The algorithm works as follows [11]

Step 0. Assigfn weights and thresholds (randomly assign little real numbers).

Step 1. Run Steps 2-9 run as long as the stopping rule is wrong.

Step 2. Run Steps 3-8 for each training data.

Step 3. Assign training data to each $x_{i} \quad(i=1,2, \ldots, n)$ input neurons.

Step 4. Calculate sum of the weighted input values for the neuron $z t_{j}(j=1,2, \ldots p)$ in the hidden layer.

$$
z t_{j}=\sum_{i=1}^{n} x_{i} v_{i j}+\theta_{j}
$$

Where, $v_{i j}$ is the weight between $i$ th neuron of input layer and $j$ th neuron of hidden layer, $\theta_{j}$ is a threshold for the $j$ th neuron of the hidden layer. To calculate the outputs of neurons of hidden layer activation function applies to $z t_{j}$.

$$
z_{j}=f\left(z t_{j}\right)
$$

Calculated results uses as an input in the next layer result send to next layer as input.

Step 5. Calculate sum of the weighted input values for the neuron $y_{k}(k=1,2, \ldots, m)$ in the output layer.

$$
y t_{j}=\sum_{j=1}^{p} z_{j} w_{j k}+\xi_{k}
$$

Where, $\omega_{j k}$ is the weight between $j$ th neuron of hidden layer and $k$ th neuron of output layer, $\xi_{\mathrm{k}}$ is a threshold for the $k$ th neuron of the output layer. To calculate the outputs of neurons of output layer activation function applies to $y t_{k}$.

$$
y_{k}=f\left(y t_{k}\right)
$$

Step 6. Compare the calculated $y_{k}$ and expected $t_{k}(\mathrm{k}=1,2, \ldots, \mathrm{m})$ for each neuron of output layer and calculate the error:

$$
\delta_{k}=\left(t_{k}-y_{k}\right) f^{\prime}\left(y t_{k}\right)
$$

Calculate the weights and thresholds changes for output layer

$$
\begin{aligned}
\Delta w_{j k} & =\alpha \delta_{k} z_{j} \\
\Delta \xi_{k} & =\alpha \delta_{k}
\end{aligned}
$$

$\alpha$ is a learning rate.

Step 7. Calculate the weights and thresholds changes for hidden layer.

$$
\begin{gathered}
\delta t_{j}=\sum_{k=1}^{m} \delta_{k} w_{j k} \\
\delta_{j}=\delta t_{j} f^{\prime}\left(z t_{j}\right) \\
\Delta v_{i j}=\alpha \delta_{j} x_{i} \\
\Delta \theta_{j}=a \delta_{j}
\end{gathered}
$$

Step 8. Calculate new values for weights and thresholds for the output layer.

$$
\begin{gathered}
w_{j k}=w_{j k}+\Delta w_{j k} \\
\xi_{k}=\xi_{k}+\Delta \xi_{k}
\end{gathered}
$$


Calculate new values for weights and thresholds for the hidden layer.

$$
\begin{gathered}
v_{j k}=v_{j k}+\Delta v_{j k} \\
\theta_{k}=\theta_{k}+\Delta \theta_{k}
\end{gathered}
$$

Step 9. Look the stopping rule.

\subsection{Back Propagation Algorithm with Adaptive Learning}

Back Propagation Algorithm with Adaptive Learning (BPAL) based on principle of updating learning rate in each iteration. Learning rate provides the addition of weight change amount in some ratio to the next update [12]. Learning rate generally is an experimentally determined constant. It takes value from $(0,1]$ interval. Therefore, regardless of the success of produced outputs the weights of the ANN model updates in the same proportion. In this study, this issue is dealt with. The change of weights is tied to the results produced by the ANN. The different learning rates have been used in each iteration. The learning rate has been established as absolute value of difference between the expected value and the calculated value as follows.

$$
\alpha=\left|t_{k}-y_{k}\right|
$$

\subsection{Acute Inflammations}

Acute Inflammations dataset has been used for implementation phase. The data sets have been obtained from the UCI Machine Learning Repository [13]. The dataset has been built by Dr. Jacek Czerniak in Systems Research Institute Polish Academy of Sciences Laboratory of Intelligent Systems in Warszawa, Poland. The data set consists of 120 data. Data has six features:

- Temperature of patient $\{35 \mathrm{C}-42 \mathrm{C}\}$

- Occurrence of nausea $\{$ yes, no $\}$

- Lumbar pain $\{$ yes, no $\}$

- Urine pushing (continuous need for urination) \{ yes, no $\}$

- Micturition pains $\{$ yes, no $\}$

- Burning of urethra, itch, swelling of urethra outlet $\{$ yes, no $\}$

There are two decisions in this dataset:

- Inflammation of urinary bladder $\{$ yes, no $\}$

- Nephritis of renal pelvis origin \{yes, no $\}$

Of these, 59 suffer with Inflammation of urinary bladder and 50 suffer with Nephritis of renal pelvis origin.

\section{Application Results}

Since dataset has two different decisions in this study two datasets have been prepared for application aim. First dataset include the six features and the decision about inflammation of urinary bladder. The second dataset contain six features and the decision about nephritis of renal pelvis origin.

\subsection{Inflammation of Urinary Bladder}

Inflammation of urinary bladder dataset has 59 patients which suffer with inflammation of urinary bladder and 61 healthy people. The dataset has been splitted into two subsets as training and testing. The training and testing dataset have 80 and 40 records respectively. First the network has been trained using BP algorithm. The three layer architecture has been used. The neurons in hidden layer have been used between $3-15$. The successful results have been obtained with the network with the five neurons in hidden layer.

Then the network has been trained using BPAL algorithm. The neurons in hidden layer have been used between $3-15$. The successful results have been obtained with the network with the fourteen neurons in hidden layer. The results of application are showed in Table I and Table II. 
TABLE I: The application result with BP algorithm for Inflammation of urinary bladder

\begin{tabular}{llll}
\hline & $\begin{array}{c}\text { Classification } \\
\text { Accuracy }\end{array}$ & Sensitivity & Specificity \\
\hline Training Set & $92.5 \%$ & $84.62 \%$ & $100 \%$ \\
Testing Set & $92.5 \%$ & $85 \%$ & $100 \%$ \\
\hline \hline
\end{tabular}

TABLE II: The application result with BPAL algorithm for Inflammation of urinary bladder

\begin{tabular}{lccc}
\hline \hline & $\begin{array}{c}\text { Classification } \\
\text { Accuracy }\end{array}$ & Sensitivity & Specificity \\
\hline Training Set & $100 \%$ & $100 \%$ & $100 \%$ \\
Testing Set & $100 \%$ & $100 \%$ & $100 \%$ \\
\hline \hline
\end{tabular}

It can be observed from Table I and II that BP algorithm reached 100\% accuracy rate for specificity, but it failed for sensitivity. However BPAL reached $100 \%$ accuracy rate for classification accuracy, sensitivity and specificity.

\subsection{Nephritis of Renal Pelvis Origin}

Nephritis of renal pelvis origin has 50 patients which suffer with nephritis of renal pelvis origin and 70 healthy people. The dataset has been splitted into two subsets as training and testing. The training and testing dataset have 80 and 40 records respectively. First the network has been trained using BP algorithm. The three layer architecture has been used. The neurons in hidden layer have been used between 3 - 15. The successful results have been obtained with the network with the eight neurons in hidden layer.

Then the network has been trained using BPAL algorithm. The neurons in hidden layer have been used between 3 - 15. The successful results have been obtained with the network with the twelve neurons in hidden layer. The results of application are showed in Table III and Table IV.

TABLE III: The application result with BP algorithm for Nephritis of renal pelvis origin

\begin{tabular}{llll}
\hline \hline & $\begin{array}{c}\text { Classification } \\
\text { Accuracy }\end{array}$ & Sensitivity & Specificity \\
\hline Training Set & $80 \%$ & $71.88 \%$ & $85.42 \%$ \\
Testing Set & $90 \%$ & $94.44 \%$ & $86.36 \%$ \\
\hline \hline
\end{tabular}

TABLE IV: The application result with BPAL algorithm for Nephritis of renal pelvis origin

\begin{tabular}{llll}
\hline \hline & $\begin{array}{c}\text { Classification } \\
\text { Accuracy }\end{array}$ & Sensitivity & Specificity \\
\hline Training Set & $100 \%$ & $100 \%$ & $100 \%$ \\
Testing Set & $100 \%$ & $100 \%$ & $100 \%$ \\
\hline \hline
\end{tabular}

It can be observed from Table III and IV that BP algorithm reached good accuracy rates, while BPAL reached $100 \%$ accuracy rate for classification accuracy, sensitivity and specificity.

\section{Conclusion}

In this study a new approach has been presented to classical Back Propagation algorithm. BPAL method has been applied to acute inflammations such as inflammation of urinary bladder and nephritis of renal pelvis origin. The results show that BPAL reaches more successful results.

\section{Acknowledgements}

This study is supported by the Scientific Research Projects Unit of Selcuk University. 


\section{References}

[1] N. Kayarvizhy, S. Kanmani, R. V. Uthariaraj, ANN Models Optimized using Swarm Intelligence Algorithms, WSEAS Transactions on Computers, Volume 13, 2014.

[2] Mukhopadhyay, S., Tang, C., Huang, J., Yu, M., \& Palakal, M. (2002). A comparative study of genetic sequence classification algorithms, neural networks for signal processing. In Proceedings of the 2002 12th IEEE workshop on 4 6 September 2002, pp. 57-66.

[3] Rumelhart DE, Hinton GE, McClelland JL. A general framework for parallel distributed processing: explorations in the microstructure of cognition, vol. 1. Cambridge: MIT Press, 1986.

[4] Huangqiong Chen, Zhigang Zeng, Deformation Prediction of Landslide Based on Improved Back-propagation Neural Network, Cognitive Computation, March 2013, Volume 5, Issue 1, pp 56-62

https://doi.org/10.1007/s12559-012-9148-1

[5] Seguritan V, Alves N Jr, Arnoult M, Raymond A, Lorimer D, Burgin AB Jr, et al. (2012) Artificial Neural Networks Trained to Detect Viral and Phage Structural Proteins. PLoS Comput Biol 8(8): e1002657. doi:10.1371/journal.pcbi.1002657

https://doi.org/10.1371/journal.pcbi.1002657

[6] R. Kottaimalai; M. P. Rajasekaran; V. Selvam; B. Kannapiran, EEG signal classification using Principal Component Analysis with Neural Network in Brain Computer Interface applications, Communication and Nanotechnology, 2013 International Conference on Emerging Trends in Computing.

[7] Hari Mohan Rai, Anurag Trivedi, Shailja Shukla, ECG signal processing for abnormalities detection using multiresolution wavelet transform and Artificial Neural Network classifier, Measurement Volume 46, Issue 9, November 2013, pages 3238-3246

https://doi.org/10.1016/j.measurement.2013.05.021

[8] Yatindra Kumar, M. L. Dewal, R. S. Anand, Epileptic seizures detection in EEG using DWT-based ApEn and artificial neural network, Signal, Image and Video Processing, October 2014, Volume 8, Issue 7, pp 1323-1334.

https://doi.org/10.1007/s11760-012-0362-9

[9] Rumelhart, D., E. ve McClelland, J.L. 1986. Parallel Distributed Processing: Explorations in the Microstructure of Cognition, Vol.1 Cambridge, MA: MIT Press.

[10] Rojas R. 1996, Neural Networks A Systematic Introduction. Springer-Verlag, Berlin, 1996.

[11]Fausett, L. 1994. Fundamentals of Neural Networks: architectures, algorithms and applications. Prentice-Hall Inc. USA.

[12] Öztemel, E., 2003. Yapay sinir Ağları. Papatya Yayınları.

[13] Lichman, M. (2013). UCI Machine Learning Repository [http://archive.ics.uci.edu/ml]. Irvine, CA: University of California, School of Information and Computer Science., last accessed February, 2016 